\title{
A SIMPLIFIED METHOD TO ASSESS THE DAMAGE OF A DEEPLY IMMERSED CYLINDER SUBJECTED TO UNDERWATER EXPLOSION
}

\author{
KEVIN BROCHARD ${ }^{1}$, HERVE LE SOURNE ${ }^{1} \&$ GUILLAUME BARRAS ${ }^{2}$ \\ ${ }^{1} \mathrm{GeM}$ Institute, UMR 6183 CNRS, ICAM Nantes, France. \\ ${ }^{2}$ Direction Générale de l'Armement - Techniques Navales, Toulon, France.
}

\begin{abstract}
The presented work is focused on the development of a simplified analytical method to study the structural response of a deeply immersed cylinder submitted to the primary shock wave of an underwater explosion. It relies on a methodology developed by Hoo Fatt and Wierzbicki where the two dimensional boundary value problem for a cylindrical shell is converted to an equivalent one-dimensional problem of a plastic string on a non-linear plastic foundation. Unstiffened cylinders immersed in shallow water have already been investigated by the authors, taking into account fluid structure interaction effects. The aim of the proposed work is to adapt the formulations to a deep immersed cylinder. The analytical developments will be presented for unstiffened cylinders. The resulting plastic dents are compared to experimental and numerical results. Although some limitations are pointed out, it is shown that this method is promising and may be advantageously used to assess rapidly the damage of a deep immersed cylinder submitted to an underwater explosions.
\end{abstract}

Keywords: fluid structure interaction, immersed cylinder, rigid-plastic analysis, Underwater explosion.

\section{INTRODUCTION}

The design of a submarine's hull is crucial for its operability and crew's safety, but also complex. Indeed, engineers need to balance lightness, acoustic discretion and resistance to both immersion pressure and environmental attacks. Submarine explosions represent a first-rate threat for the integrity of the hull, whose behavior needs to be properly analyzed. This paper is the follow-up to two previous papers from the authors [1] and [2]. In these papers, the string-on-foundation model, developed originally by Wierzbicki and Hoo Fatt [3], is coupled to a simplified modelling of fluid-structure interaction between the shell and the surrounding fluid, but without taking into account the effects of hydrostatic pressure. This methodology is then validated by comparison with numerical and experimental results, and its limitations are highlighted. However, Gupta [4] and Biglarkhani and Sadeghi [5] have shown experimentally and numerically that the hydrostatic pressure has a dramatic influence on the mechanical behavior of the cylinder, and may lead to considerable damage even if the explosion is weak. This paper presents an extension of the presented methodology in [2] in order to take into account the effects of hydrostatic pressure, based on the formulations presented by Wierzbicki and Suh [6]. Closed-form expression of the final deflection of a deeply immersed cylinder subjected to the primary shock wave of an underwater explosion are obtained and compared to numerical results.

\section{EXPLOSIVE LOADING}

A simplified modelling of the primary shock wave loading has been developed and is presented in a previous paper [2]. In this paper, only the major outcome are addressed. 


\subsection{Theoretical foundations}

Consider a cylindrical shell of length $L$, radius $R$ and thickness $h$, clamped at both ends. It is filled with air and fully immersed in water at a depth $D_{i m}$. An explosive charge of mass $C$ detonates at a distance $D_{0}$ from the stand-off point $S_{0}$, located at the intersection of cylinder's planes of symmetry, as shown in Fig. 1. On a current point $S$ of the shell, shock wave's pressure amplitude may be calculated with the following expressions proposed by Cole [7]:

$$
p_{I}(S \mid t)=P_{S} e^{-t / T_{S}}
$$

With,

$$
P_{S}=K_{P}\left(C^{1 / 3} / D\right)^{A_{P}}, T_{S}=K_{T} C^{1 / 3}\left(C^{1 / 3} / D\right)^{A_{T}} .
$$

Here, $D$ is the distance between $S$ and the charge centre location and $\mathrm{K}_{\mathrm{p}}, \mathrm{K}_{\mathrm{T}}, \mathrm{A}_{\mathrm{p}}$ and $\mathrm{A}_{\mathrm{T}}$ are characteristic parameters of the explosive. In the following developments, the fluid is supposed to be infinite, inviscid and incompressible so that a potential flow can be assumed. In a point far enough from the charge, the pressure field in the water also verifies Bernoulli linear equation.

When the shockwave generated by the underwater explosion impacts the shell, fluid-structure interactions lead to a pressure field in the fluid of the following form:

$$
p(P \mid t)=p_{I}(P \mid t)+p_{S}(P \mid t) .
$$

Where $p_{S}$ is the so-called scattered pressure, i.e. the sum of wave reflected on the fixed and rigid cylinder and the wave radiated by shell deformation. It constitutes an unknown of the problem.

\subsection{Simplified explosive loading}

The pressure loading acting on the shell is broken down into two phases, in a similar way to the one used by Geers [8]. During the first phase, the shell is supposed to remain rigid and by considering the "short time approximation", a kinetic energy transmitted to the cylinder by the shock wave is calculated. During the second phase, the cylinder deforms and, according
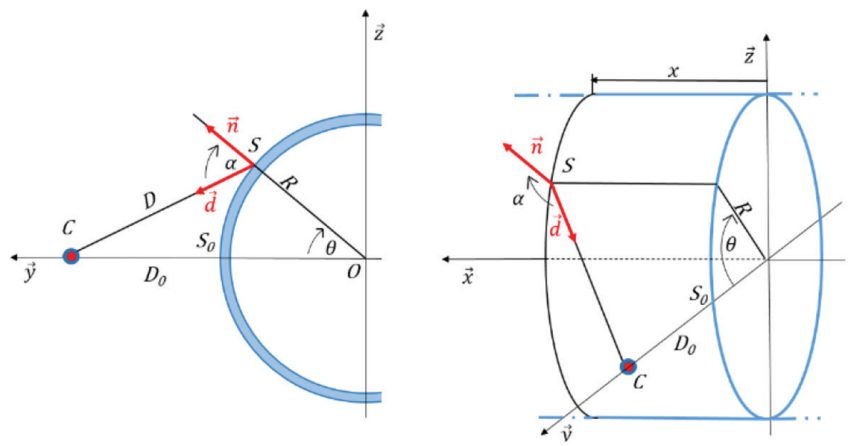

Figure 1: Initial configuration of an immersed cylinder subjected to an underwater explosion. 
to the "long-time approximation", the related shell displacements create an additional pressure loading.

\subsubsection{First phase of loading}

During this very short phase, the deformation of the shell is supposed to be sufficiently small to be neglected so only inertia forces are considered, as shown by Taylor [9]. Conservation of momentum expressed at a point $S$ of the shell thus gives:

$$
\frac{\partial \dot{w}}{\partial t}+\frac{\rho_{f} c_{f}}{m} \dot{w}(t)=\frac{2 P_{S}}{m} e^{-t / T_{S}} .
$$

Where $\dot{w}(t)$ is the radial shell velocity at point $S$ and $m$ is the mass per unit area of the shell. $P_{S}$ and $T_{S}$ are calculated for each point $\mathrm{S}$ with help of eqns (2). By introducing the following dimensionless parameter $\beta_{f}=\rho c T_{S} / \mathrm{m}$ is the ratio of the mass of water displaced by the shock wave in the direction normal to the shell surface to the mass of the shell at point $S$, per unit area. Eqn (4) is a simple first order linear differential equation whose resolution is detailed in [2]. A field of maximal velocity attained by the shell velocity $V_{m}(S)$ is obtained and can be estimated with the following expression:

$$
V_{m}(S)=\frac{2 P_{S} T_{S}}{m} \beta_{f}{ }^{\frac{\beta_{f}}{1-\beta_{f}}}\left(\frac{1+\cos \alpha}{2}\right) .
$$

Where the term $(1+\cos \alpha) / 2$ is used to take into account the effect of the shock wave incidence. This velocity field is then used to determine the total kinetic energy $E_{c}$ which has been transmitted by the shock to the cylinder shell at the end of the first phase of loading:

$$
E_{c}=\frac{1}{2} \int_{S_{0}} m V_{m}(S)^{2} d S_{0}
$$

\subsubsection{Second phase of loading}

During the second phase of loading, mechanical deformation of the shell takes place and produces an additional pressure loading proportional to the shell deceleration. Moreover, since $\theta_{d} \gg T_{S}$, the incident pressure field related to the shock wave has vanished, i. e. $p_{I}(S \mid t) \approx 0$. The fluid structure interaction is thus assumed to be limited to water inertial effects. In reality, as mentioned by Taylor [9], the radiated pressure field is driven by velocity continuity between fluid-structure interface so radiation damping may still persist after pressure decay. However, in order to simplify the analytical derivation of the shell movement equation, such damping is only considered in the first phase of loading and conservatively ignored in the second phase. Finite element analyses of an immersed cylinder subjected to an underwater explosion, carried out during this research work and validated by underwater explosion experiments, show that the shell deforming region is elliptic, as presented in Fig 2(a). Its circumferential extent then varies as a function of the position $x$ along the cylinder generator. The added mass of water may be derived by postulating the following approximate displacement field for the shell deforming region:

$$
w(S \mid t)=\left\{\begin{array}{cc}
0, & t<0 \\
\delta(t) f(x) g(\theta), & t>0
\end{array}\right.
$$




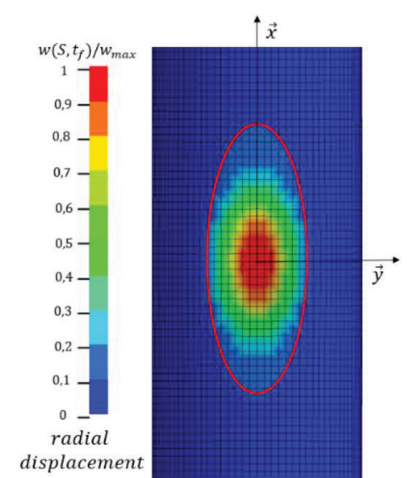

a)

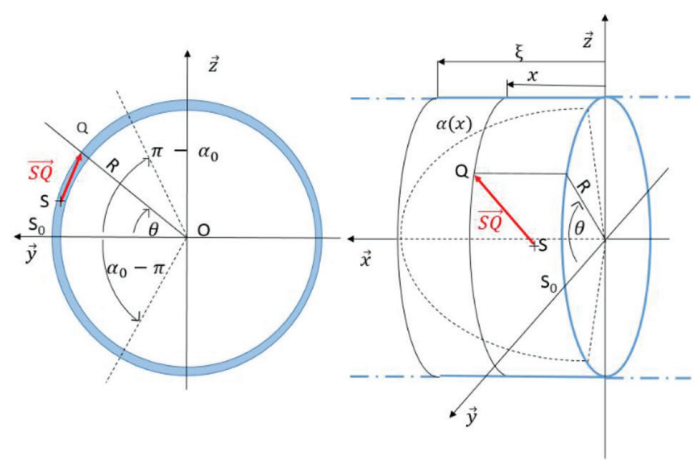

b)

c)

Figure 2: Notations for added mass of water calculation.

Where $f$ and $g$ are shape functions respectively along longitudinal and circumferential directions, chosen from underwater explosion tests and numerical analyses. Considering that $p_{I}(S \mid t) \approx 0$, the pressure at current point $S$ is:

$$
p(S \mid t) \approx-\ddot{\delta}(t) \frac{\rho_{f}}{2 \pi} \int_{-\xi}^{\xi} \int_{-\alpha(x)}^{\alpha(x)} \frac{R f(x) g(\theta)}{\overline{\|S Q\|}} d \theta d x .
$$

Where $2 \xi$ is the longitudinal extent of shell plastic deformation and $\alpha(x)=\left(\pi-\alpha_{0}\right) \sqrt{1-(x / \xi)^{2}}$, which is the equation of an ellipse (see Fig $3 b$ and c). The water added mass per unit area at point $S$ writes:

$$
m_{a}(S)=\frac{\rho_{f}}{2 \pi} \int_{-\xi}^{\xi} \int_{-\alpha(x)}^{\alpha(x)} \frac{R f(x) g(\theta)}{\|S Q\|} d \theta d x .
$$

At each point $S$, expression (10) is integrated numerically and a field of water added masses distributed on the shell deformed area is obtained. In order to simplify the mathematical developments, this field may be expressed as:

$$
m_{a}(x, \theta)=M_{a} h_{1}(x) h_{2}(\theta) .
$$

Where $x, \theta$ are the point $S$ cylindrical coordinates and $M_{a}$ is the added mass of water per unit area evaluated at stand-off point $S_{0}$. Shape functions $h_{1}$ and $h_{2}$ may be extracted from the actual water added mass field evaluated by numerical integration of eqn (9) as mentioned before. In our study, $h_{1}$ is a third order polynomial function and $h_{2}$ a fourth order one, whose expressions are detailed in [2].

\section{CYLINDRICAL SHELL MECHANICAL BEHAVIOUR}

The extension of rigid-plastic string-on-foundation model, developed in our previous paper [2], in order to take into account the effects of the hydrostatic pressure is presented in this section. Consider the sealed cylinder depicted in Fig. 3, it is clamped at its extremities, except in the longitudinal direction $\vec{x}$. Firstly, the elastic energy stored in the cylinder is calculated. 


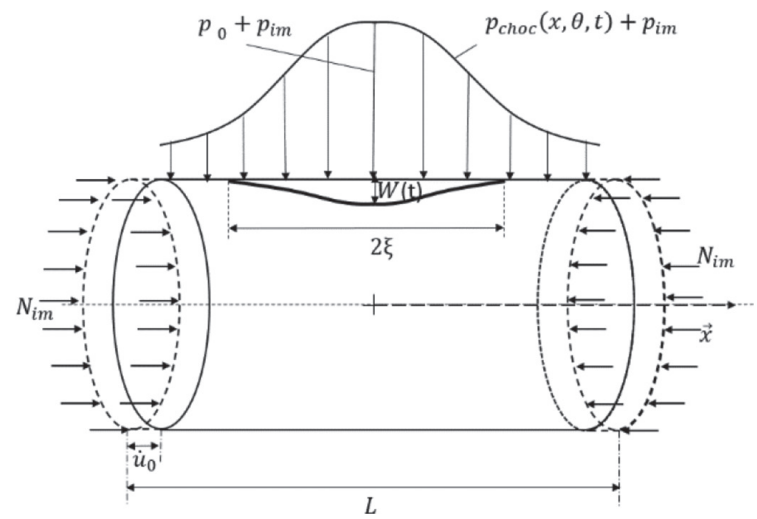

Figure 3: Deeply immersed cylinder impacted laterally by the primary shock wave of an underwater explosion.

Secondly, a mechanical model of the cylinder's response to the primary shock wave is developed, which includes both axial and radial component of the hydrostatic pressure. Inclusion of these effects in the model is based on the method developed by Wierzbicki and Suh [6] to study the indentation of a tube under combined loadings.

Using the membrane theory of shells, the elastic energy stored by the cylindrical shell under the action of the hydrostatic pressure $p_{i m}=\rho g D_{i m}$ is calculated with the following expression:

$$
W_{E}=\int_{-L}^{L} \int_{-\pi}^{\pi} \frac{E h}{2\left(1-v^{2}\right)}\left(\epsilon_{x}{ }^{2}+\epsilon_{\theta}{ }^{2}+2 v \epsilon_{x} \epsilon_{\theta}\right) d x a d \theta .
$$

Using the well-known expressions of axial and circumferential strains $\epsilon_{x}=-0.2 p_{i m} R / E h$ and $\epsilon_{\theta}=-0.85 p_{i m} R / E h$ and integrating eqn (11) allows to obtain a closed-form expression of the elastic deformation energy:

$$
W_{E}=\frac{(0.76+0.17 v)}{E h\left(1-v^{2}\right)} 2 \pi L p_{i m}{ }^{2} R^{3} .
$$

The loading due to the underwater explosion shock wave and immersion pressure is supposed to be severe enough so that the shell deforms plastically. As a result, the shell undergoes radial deflection $w(x, \theta, t)$, where $x, \theta$ denote axial and circumferential coordinates respectively and $t$ is the time. In the range of moderately large deflection, elastic deformations are negligible as compared to plastic ones. Therefore, the material is assumed to be isotropic, time-independent, and rigid-perfectly plastic, described by a flow stress $\sigma_{0}$. The overall equilibrium is expressed via the principle of virtual velocities in shell coordinates:

$$
2 N_{i m} \dot{u}_{0}+\int_{S_{0}} p \dot{w} d S_{0}-\int_{S_{0}}(m \ddot{w}) \dot{w} d S_{0}=h \int_{S_{0}} \sigma_{i j} \dot{\epsilon_{i j}} d S_{0}
$$

Where $\sigma_{i j}$ and $\epsilon_{i j}$ are the components of stress and strain rate tensors and $d S_{0}=d x R d \theta$. Right hand side term of eqn (13) is the rate of energy dissipated by plastic deformation of the 
shell and the left hand side term corresponds to the rate of external work. The first term corresponds to the work rate of the axial force due to immersion pressure. The third one is related to the inertial forces associated to the shell radial acceleration and the second describes the work rate of the lateral pressure which can be expressed as:

$$
p(x, \theta, t)=p_{\text {choc }}(x, \theta, t)+p_{\text {im }} .
$$

Where $p_{\text {choc }}$ is the pressure obtained from eqn (8). Using the simplified expression of the field of water added mass given by eqn (10), and assuming a plane stress state and the Love-Kirchhoff hypothesis for the shell deformation, eqn (14) becomes:

$$
2 N_{i m} \dot{u}_{0}+\int_{S_{0}} p_{i m} \dot{w} d S_{0}-\int_{S_{0}}\left(m \ddot{w}+m_{a}(x, \theta) \ddot{w}\right) \dot{w} d S_{0}=\int_{S_{0}}\left(N_{\alpha \beta} \dot{\epsilon}_{\alpha \beta}+M_{\alpha \beta} \dot{\kappa}_{\alpha \beta}\right) d S_{0} .
$$

Where $\dot{\epsilon}_{\alpha \beta}$ and $\dot{\kappa}_{\alpha \beta}$ are the generalized strain and curvature rate tensors, and $N_{\alpha \beta}$ and $M_{\alpha \beta}$ are the corresponding tensors of the membrane force and bending moment. So right-hand side term of eqn (15) is the rate of internal energy $\dot{W}_{\text {int }}$. For relatively thin shells, i.e. when $20<R / h<150$, undergoing moderately large deflection, i.e. when $W / R<0.2$, some deformation modes such as transverse shearing may be neglected. Considering all hypotheses detailed in references [1] and [2], the rate of internal energy writes:

$$
\dot{W}_{i n t}=\int_{S_{0}}\left(\left|M_{p l} \dot{\kappa}_{\theta \theta}(x, \theta, t)\right|+\left|N_{p l} \dot{\varepsilon}_{x x}\right|\right) d S_{0} .
$$

Where $M_{p l}=\sigma_{0} h^{2} / 4$ is the fully plastic bending moment per unit length and $N_{p l}=\sigma_{0} h$. The two terms on the right hand side of eqn (16) represent respectively the rate of bending energy in the circumferential direction (crushing of rings) and the rate of axial membrane energy (stretching of generators). In the presented model, these two deformation modes are assumed to be decoupled, which simplifies the mathematical resolution of the problem. Physically, this means that the cylinder is considered as a set of independent generators supporting independent rings, which may deform independently without shearing. Replacing eqn (16) into eqn (15), the formulation of the problem may be written as:

$$
2 N_{i m} \dot{u}_{0}+\int_{S_{0}} p_{i m} \dot{w} d S_{0}=\int_{S_{0}}\left[\left|M_{p l} \dot{\kappa}_{\theta \theta}(x, \theta, t)\right|+\left|N_{p l} \dot{\varepsilon}_{x x}\right|+\left(m \ddot{w}+m_{a}(x, \theta) \ddot{w}\right) \dot{w}\right] d S_{0} .
$$

\section{CYLINDER RESPONSE CONSIDERING AXIAL HYDROSTATIC PRESSURE}

In order to calculate the contribution of the deforming generators, the global strain rate $\dot{\varepsilon}_{x x}$ is decomposed into two parts:

$$
\int_{0}^{\xi} \dot{\varepsilon}_{x x} d x=\int_{0}^{\xi}\left[\dot{\varepsilon}_{1}+\dot{\varepsilon}_{2}\right] d x
$$

The strain rate $\dot{\varepsilon}_{1}$, related to local indentation, differs from one generator to another. The strain rate $\dot{\varepsilon}_{2}$, due to uniform compression of the tube is the same for all generators. According to the theory of moderately large deflections of beams, one has:

$$
\dot{\varepsilon}_{1}=w^{\prime} \dot{w} .
$$


Where the prime denotes derivation with respect to $x$. Considering the displacement field given by eqn (7) and taking $f=\left(1-(x / \xi)^{2}\right)^{2}$, the contribution of a single generator to the rate of energy dissipated through radial deformation writes:

$$
2 N_{p l} \int_{0}^{\xi} \dot{\varepsilon}_{1} d x=2 N_{p l} g^{2} \delta \dot{\delta} \int_{0}^{\xi}\left(f^{\prime}\right)^{2} d x=\frac{256 N_{p l}}{105} g^{2} \frac{\delta \dot{\delta}}{\xi}
$$

The pure axial strain rate, which results from the beam-like theory, is linear $\dot{\varepsilon}_{2}=\ddot{u}$, and can be readily integrated with respect to $x$. The rate of energy dissipated by all the deforming generators $\dot{W}_{m}$ is then:

$$
\dot{W}_{m}=4 R N_{p l}\left[\int_{0}^{\pi-\alpha_{0}}\left|\frac{\delta \dot{\delta}}{\xi} \frac{128}{105} g^{2}+\dot{u}_{0}\right| d \theta+\int_{\pi-\alpha_{0}}^{\pi}\left|\dot{u}_{0}\right| d \theta\right] .
$$

Where $\dot{u}_{0}=\dot{u}(\xi)$ and $\pi-\alpha_{0}$ is the half-circumferential extent of the deformed area, which is supposed to be rectangular. In the following developments, $\alpha_{0}$ will be set to zero, so the circumferential extent of the deformed area is equal to whole circumference of a cylinder cross section. Wierzbicki and Suh [6] chose the same circumferential extent for their model. In previous research [2], a sixth-order polynomial function was chosen for shape function. A closed-form expression of the integral in eqn (21) may be obtained by introducing a simplified expression of $g^{2}$ :

$$
\tilde{g}(\theta)=\left\{\begin{array}{cc}
(1-\theta / a \pi) \dagger, \theta \leq a \pi \\
0, \quad \theta>a \pi
\end{array}\right.
$$

Where $a$ may vary from 0 to 1 . Functions $g, g^{2}$ and $\tilde{g}$ are plotted in Fig 4 .

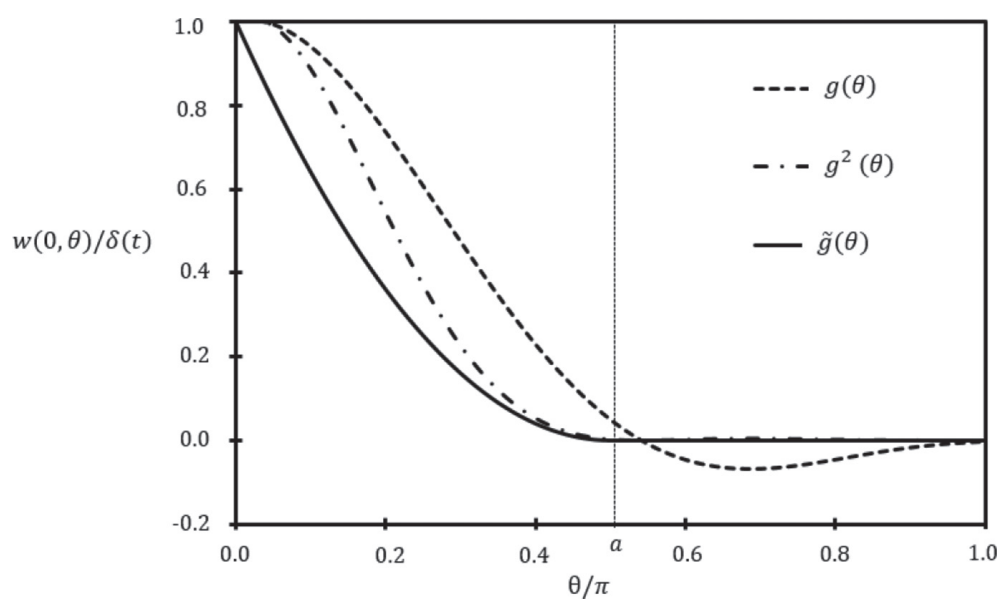

Figure 4: Plots of displacement shape functions along circumferential direction. 
By setting $\theta=\left(\pi-\alpha_{0}\right) \tilde{\theta}$ and taking $\alpha_{0}=0$, eqn (22) becomes:

$$
\dot{W}_{m}=4 \pi R N_{p l}\left[\int_{0}^{a}\left|\frac{\delta \dot{\delta}}{\xi} \frac{128}{105}(1-\tilde{\theta} / a) \dagger+\dot{u}_{0}\right| d \tilde{\theta}+\int_{a}^{1}\left|\dot{u}_{0}\right| d \tilde{\theta}\right]
$$

In absence of radial deformation, $\dot{\kappa}_{\theta \theta}=\dot{w}=0$ in eqn (18), which leads to:

$$
N_{i m}=-N_{p}
$$

Where $N_{p}=2 \pi R N_{p l}$ is the plastic force capacity of an undeformed cylinder cross-section. This means that the cylinder remains free of axial deformation as long as the axial hydrostatic force remains lower than $\left|N_{p}\right|$. As observed by Wierzbicki and Suh [6], the axial force acting alone may not lead to the cylinder failure. However, it may dramatically change the denting strength of the pre-stressed shell. When the shell radial deformation is non zero, the total axial force in a cross-section may be written as:

$$
N_{i m}=2 \pi R N_{p l}\left[\int_{0}^{a} \operatorname{sign}\left(\frac{\delta \dot{\delta}}{\xi} \frac{128}{105}(1-\tilde{\theta} / a) \dagger+\dot{u}_{0}\right) d \tilde{\theta}+\int_{a}^{1} \operatorname{sign}\left(\dot{u}_{0}\right) d \tilde{\theta}\right]
$$

The argument of the sign function in the first integrand of eqn (26) changes its sign when $\tilde{\theta}=\tilde{\theta}_{1}$ such as:

$$
\frac{\delta \dot{\delta}}{\xi} \frac{128 N_{p l}}{105}\left(1-\tilde{\theta}_{1} / a\right)^{2}=-\dot{u}_{0}
$$

After integration, eqn (25) becomes:

$$
N_{i m}=-N_{p}\left(1-2 \tilde{\theta}_{1}\right)
$$

The axial force $N_{i m}$ is also related to the immersion depth $D_{i m}$ :

$$
N_{i m}=-\pi R^{2} p_{i m}=-\rho g \pi R^{2} D_{i m} .
$$

According to Wierzbicki and Suh [6], eqn (27) means that for any choice of $D_{i m}$ such as $-N_{p}<N_{i m}<0$, there is a unique point on the cylinder half-circumference $\tilde{\theta}=\tilde{\theta}_{1}^{i m}$ at which axial strain rate vanishes and stresses change from tension to compression. Having determined such switching point, integration of eqn (24) can be performed to give:

$$
\dot{W}_{m}=\frac{4 \pi R N_{p l}}{3} \frac{\delta \dot{\delta}}{\xi} \frac{128}{105} a\left(1-\frac{1}{4 a^{3}}\left(2 a-1-\frac{N_{i m}}{N_{p}}\right)^{3}\right)+2 N_{i m} \dot{u}_{0}
$$


Disregarding the axial hydrostatic force and following the same procedure, the energy dissipated by the deforming generator $\dot{W}_{\tilde{m}}$ can be calculated as follows:

$$
\dot{W}_{\tilde{m}}=\frac{4 \pi R N_{p l}}{3} \frac{\delta \dot{\delta}}{\xi} \frac{128}{105} a .
$$

In the following development, $a$ is set to 0.5 to be in accordance with the choice of circumferential shape function. The second term in the right hand-side of eqn (29) is identical to the first term of the left-hand side of eqn (17). The second term in eqn (17), corresponding to the work rate of the radial pressure of immersion can be expressed as a function of $\dot{\delta}$ :

$$
4 R \int_{0}^{\pi} \int_{0}^{\xi} p_{i m} \dot{\delta} f(x) g(\theta) d x d \theta=\frac{8 \xi}{15} \bar{p}_{i m} \dot{\delta}
$$

Here, $\quad \bar{p}_{i m}=2 \pi R p_{i m} G_{i m}$ is the equivalent radial immersion pressure where $G_{i m}=\int_{0}^{\pi} g(\theta) d \theta=0.19$ is obtained by numerical integration. This equivalent parameter can be interpreted as an integrated average value of the respective quantity with the associated velocity as a weighing function. Concerning other terms of eqn (17), detailed derivations of their expressions with respect to $\dot{\delta}$ may be found in [1]. For the time being, the water inertial effects are not considered, so $m_{a}(x, \theta)=0$. Replacing these expressions in eqn (17), a second order linear ordinary differential equation is obtained:

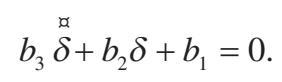

Where coefficients $b_{1}, b_{2}$ and $b_{3}$ are defined as:

$$
b_{1}=\frac{8 \xi}{15}\left(\bar{q}-\bar{p}_{i m}\right) ; b_{2}=\frac{128 \bar{N}}{105 \xi}\left(1-\frac{1}{4 a^{3}}\left(2 a-1-\frac{N_{i m}}{N_{p}}\right)^{3}\right) ; b_{3}=\frac{128 \xi \bar{m}}{315}
$$

Here, $\bar{q}=2 M_{p l} G_{q} / R$ is the equivalent ring crushing resistance per unit length, $\bar{N}=2 \pi R N_{p l}(2 a / 3)$ is the equivalent tensile force per unit length and $\bar{m}=2 \pi R m G_{m}$ is the equivalent mass per unit length, where $G_{q}=6.3$ and $G_{m}=0.22$ are also determined by numerical integration. Initial conditions related to eqn (32) may be written as follows:

$$
\delta(0)=\delta_{i} \& \dot{\delta}(0)=\dot{\delta}_{i}
$$

The initial velocity $\dot{\delta}_{i}$ is calculated by considering the kinetic energy $E c$ transmitted to the cylinder wall by the shock wave, at the end of first phase of loading (details of derivation of $\dot{\delta}_{i}$ are given in [2]):

$$
\dot{\delta}_{i}=\sqrt{E_{c} / \frac{128 \xi \bar{m}}{315}} .
$$


The initial displacement $\delta_{i}$ is determined from the cylinder hydrostatic pre-loading, by converting the corresponding elastic energy stored inside the structure into plastic energy to be dissipated through the plastic mechanism. An « equivalent » initial deflection is thus calculated by solving the following equation:

$$
\frac{256 \bar{N}}{105 \xi} \delta_{i}^{2}+\frac{16 \bar{q} \xi}{15} \delta_{i}-W_{E}=0 .
$$

Above equation admits the following solution:

$$
\delta_{i}=\frac{-16 \bar{q} \xi / 15^{+\sqrt{\Delta}}}{256 \bar{N} / 105 \xi} \text { with } \Delta=\left(\frac{16 \bar{q} \xi}{15}\right)^{2}+4 W_{E} \frac{256 \bar{N}}{105 \xi}
$$

Once initial conditions $\delta_{i}$ and $\dot{\delta}_{i}$ are known, the solution of eqn (32) writes:

$$
\delta(t)=\delta_{i} \cos \left(\sqrt{b_{2} / b_{3}} t\right)+\frac{b_{1}}{b_{2}}\left(\cos \left(\sqrt{b_{2} / b_{3}} t\right)-1\right)+\dot{\delta}_{i} \sqrt{b_{3} / b_{2}} \sin \left(\sqrt{b_{2} / b_{3}} t\right)
$$

The constitutive material being considered as rigid-plastic, the cylinder is supposed to deform without any elastic restoring movement. The permanent deflection amplitude is thus defined by $\delta_{f}=\delta\left(t=t_{f}\right)$ where $t_{f}$ is the time at which the central velocity vanishes. By differentiating eqn (48) and by setting $\dot{\delta}\left(t_{f}\right)=0$, a relation is found for deriving $t_{f}$ :

$$
\tan \left(\sqrt{b_{2} / b_{3}} t_{f}\right)=\frac{\dot{\delta}_{i}}{\sqrt{b_{2} / b_{3}\left(b_{1} / b_{2}+\delta_{i}\right)}}
$$

Eqn (39) is solved for both values of parameter $b_{2}$, i.e. with and without considering axial forces, for a charge of mass $C=1.1 \mathrm{~kg}$ at a distance $D_{0}=0.42 \mathrm{~m}$, and a cylinder described in Table 1. Fig 5 shows the discrepancies between both solutions, for a depth of immersion $\mathrm{D}_{\text {im }}$ ranging from 1 to $350 \mathrm{~m}$. In this figure, analytical values are compared to values obtained from numerical simulations, carried out using nonlinear finite element solver LS-DYNA coupled with code USA. Detailed description of the simulation methodology is available in
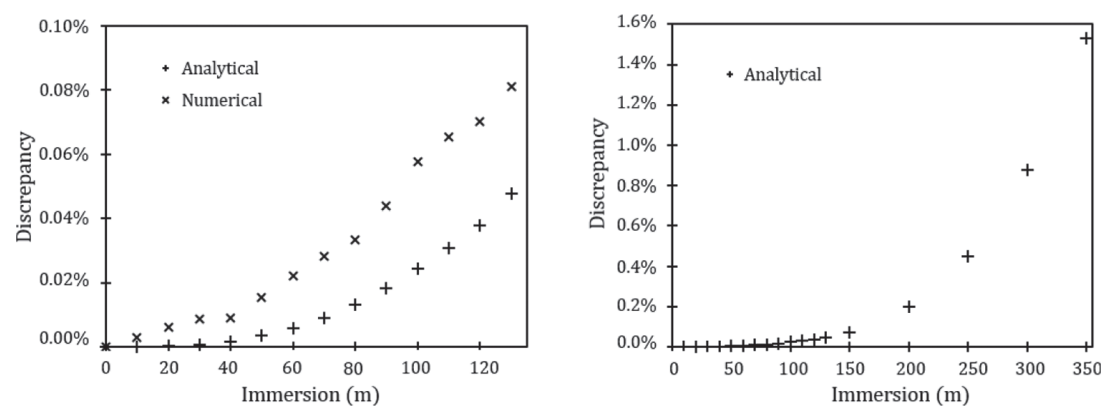

Figure 5: Discrepancy in final deflection $\delta_{f}$ between model with and without axial forces. 
[1] and [2]. Analytical discrepancies is approximatively three times to twice as small as numerical ones for the considered depths of immersion. Analytical discrepancies remain small, inferior to $1.6 \%$ for immersion depths up to $350 \mathrm{~m}$, which is quite closed to the critical immersion of the cylinder, equal to $487 \mathrm{~m}$ according to formulations from Pinna [10]. In conclusion, this simplified mechanical model shows that axial forces may be neglected when estimating the response of an immersed cylinder to an underwater explosion. A more complex mechanical is then developed, similar to the one presented in [2], which includes only the radial pressure of immersion.

\section{VALIDATION OF THE METHOD INCLUDING IMMERSION EFFECTS}

\subsection{Mechanical model}

This new mechanical is developed from eqn (17), but this time the deformed area is considered elliptic, i.e. circumferential extent is varying with respect to $x$, with $\alpha(x)=\left(\pi-\alpha_{0}\right) \sqrt{1-(x / \xi)^{2}}$. Added mass of water is also considered, and modeled with the simplified field described by eqn (10). With these assumptions, eqn (17) can be expressed as a second ordinary linear differential equation:

$$
a_{3} \ddot{\delta}+a_{2} \delta+a_{1}=0 .
$$

Where coefficients $a_{1}, a_{2}$ and $a_{3}$ are defined as:

$$
\begin{gathered}
a_{1}=\frac{3 \xi}{16\left(1-\frac{\alpha_{0} / \pi}{\pi}\right)} \bar{q}-\frac{5 \pi \xi}{32} \vec{p}_{i m} ; a_{2}=\bar{N}^{\prime} \frac{\pi}{\xi}\left(1-\frac{\alpha_{0}}{\pi}\right) \frac{5 \pi}{16} ; \\
a_{3}=\left(\vec{m}+\bar{m}_{a}^{\prime} G_{m a}^{\prime}\right) \pi \xi\left(1-\frac{\alpha_{0}}{\pi}\right) \frac{63 \pi}{512} .
\end{gathered}
$$

Where $\vec{p}_{i m}^{\prime}=2 R\left(\pi-\alpha_{0}\right) p_{i m} G_{i m}, \quad \vec{N}^{\prime}=2 R N_{p l} G_{m}, \quad \bar{m}^{\prime}=2 R m G_{m}$ and $\bar{m}_{a}^{\prime}=2 R m_{a} G_{m a}$. Coefficients $G_{m a}=\int_{0}^{1} h_{2}(\tilde{\theta}) g^{2}(\tilde{\theta}) d \tilde{\theta}=0.82 G_{m}$ and $G_{m a}=\int_{0}^{\xi} h_{1}(x) f^{2}(x) d x=0.92$ are obtained from numerical integration. In our previous article [2], it has been shown that it is necessary to take into account the deformed area evolution with respect to time, in order to correctly estimate the shell final deflection. It is done by considering a linear variation of damaged parameters, which leads to the determination of averaged values for coefficients $a_{i}$, given by the following equation:

$$
\tilde{a}_{i}=\frac{1}{\left(\xi_{\max }-\epsilon_{1} \xi_{\max }\right)\left(\alpha_{0 \max }-\epsilon_{2} \pi\right)} \int_{\epsilon_{1} \xi_{\max }}^{\xi_{\max }} \int_{\epsilon_{2} \pi}^{\alpha_{0 \max }} a_{i}\left(\xi, \alpha_{0}\right) d \alpha_{0} d \xi
$$

Where $\epsilon_{1} \xi_{\max }$ and $\epsilon_{2} \pi$ are respectively longitudinal and circumferential initial damaged lengths. Resulting expression for coefficients $\tilde{a}_{i}$ are detailed in [2]. Regarding initial 
conditions related to eqn (40), determination of $\delta_{i}$ and $\dot{\delta}_{i}$ follows the same procedure. Solution to eqn (40) is identical to the one of eqn (32), but with coefficients $\tilde{a}_{i}$ instead of coefficients $b_{i}$.

\subsection{Comparison with finite element results}

In order to validate the model presented in previous section, obtained results are compared with numerical results considered as a reference. For that purpose, numerical simulations of the problem depicted in Fig. 3 have been carried out using the Underwater Shock Analysis (USA) code coupled with nonlinear finite element solver LS-DYNA. The response of the cylinder submitted to underwater explosion is simulated up to $50 \mathrm{~ms}$. An elastic-plastic behavior law with strain hardening is considered to simulate the behavior of the stainless steel which constitutes the cylinder. Table 1 sums up shell characteristics as well as material properties. The same charge as in section 4 is used, and the considered depths of immersion range from $1 \mathrm{~m}$ to $130 \mathrm{~m}$ with steps of $10 \mathrm{~m}$.

Simulations of these scenarios have been conducted using the simplified model with two different yield criteria; one inscribing the actual one: $\sigma_{\text {inscr. }}=0.63 \sigma_{0}$, and one circumscribing it: $\sigma_{\text {circum }}=\sigma_{0}$. As shown in Fig 6(a), the analytical model overestimates the energy dissipated plastically when compared to numerical simulations. The difference decreases with the increase in immersion. Regarding stored elastic energy, Fig 6(b) proves that the analytical model is in accordance with numerical results, with discrepancies always below $5 \%$.

Final deflections $\delta_{f}$ obtained from numerical simulations and simplified method are compared in Fig. 7(a), for all depths of immersion. The increase in deflection $\Delta \delta_{f}=\delta_{f}\left(D_{i m}\right)-\delta_{f}(0)$ is also plotted in Fig. 7(b). Fig. 7(a) shows that the actual response of the cylinder lays between solutions obtained with inscribing and circumscribing yield

Table 1: Characteristics of cylinder and material.

\begin{tabular}{llll}
\hline Cylinder & \multicolumn{3}{l}{ Material } \\
\hline Length $L(\mathrm{~m})$ & 2.8 & Young Modulus $(\mathrm{Pa})$ & $2 \mathrm{E} 11$ \\
Radius $R(\mathrm{~m})$ & 0.7 & Density $\left(\mathrm{kg} \cdot \mathrm{m}^{-3}\right)$ & 7800 \\
Thickness $h(\mathrm{~m})$ & 0.015 & Yield Stress $(\mathrm{MPa})$ & 355 \\
& & Tangent Modulus $(\mathrm{MPa})$ & 415 \\
\hline
\end{tabular}
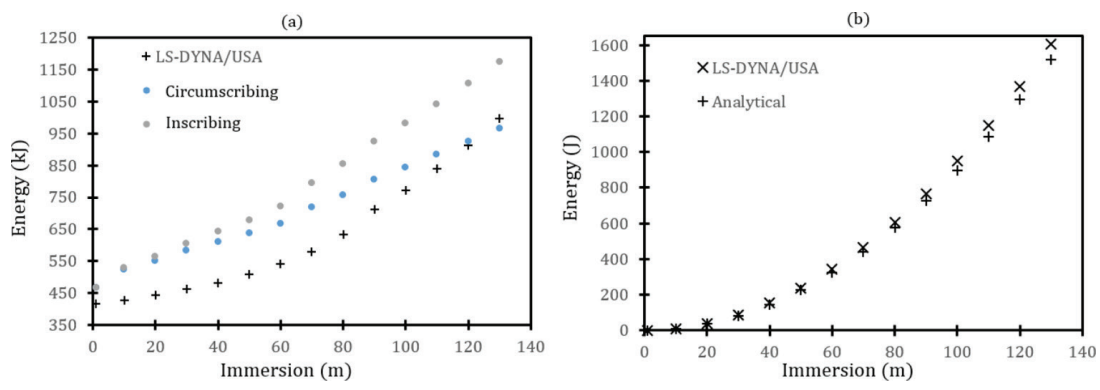

Figure 6: Comparison of analytical and numerical results : (a) Plastically dissipated energy (b) Stored elastic energy. 
(a)

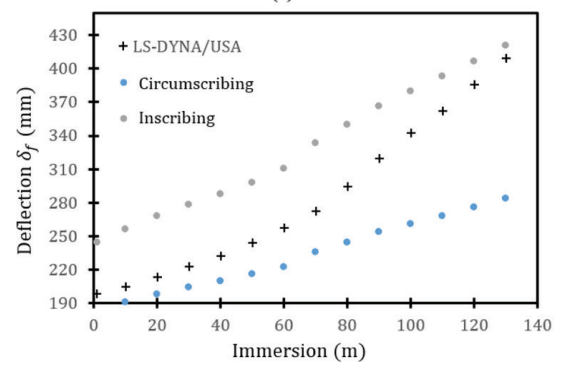

(b)

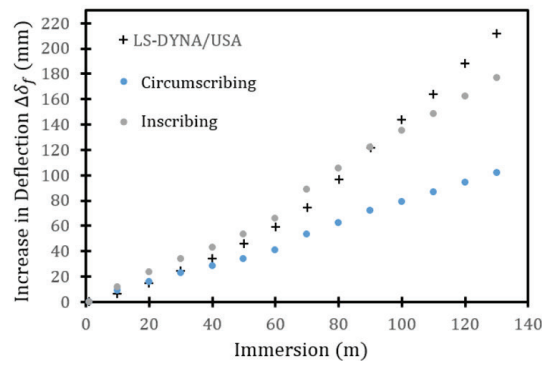

Figure 7: Comparison of analytical and numerical results : (a) Shell final deflection $\delta_{f}-$ (b) Increase in shell final deflection $\Delta \delta_{f}$.

criteria, with an error inferior to $30 \%$ whatever the depths of immersion. Concerning the increase in deflection, analytical solutions follow the same trend as the numerical solutions up to a depth of immersion of $70 \mathrm{~m}$. For deeper immersion, the actual increase in deflection is greater than the analytical ones. This highlights the limitation of the simplified method, for which a new mechanism should be developed for the response of the shell to the radial immersion pressure. In fact, there is a change in the mechanical behaviour of the shell at some point of the cylinder's response to the combined loading of the shockwave and the hydrostatic pressure.

\section{CONCLUSION}

In the present paper, a simplified method for assessing the damage of a deeply immersed cylinder subjected to underwater shock is described for application to pre-design analysis. The method is based on a simplified mechanism of shell deformation, the so-called plastic string-on-foundation mechanism, together with an approximation of the primary shock wave loading from the underwater explosion. It has been shown that the axial forces applied by the hydrostatic pressure on the cylinder are of little influence on the response of the shell. However, radial pressure may have a dramatic effect on the survivability of a cylinder impacted by a shock wave. The developed simplified method approximate quite well the behavior of the shell for moderate depth of immersion, but a new mechanism of deformation of the shell should be developed in order to consider deeper immersion. The simplified method should also be extended to ring-stiffened shell, and it will be the subject of future research.

\section{ACKNOWLEDGEMENTS}

The authors would like to thank "DGA Naval Systems" for its financial support and technical participation in defining the scope of this work.

\section{REFERENCES}

[1] Brochard, K., Le Sourne, H. \& Barras, G., A simplified method to assess the damage of an immersed cylinder subjected to underwater explosion. Proceedings of the 6th International Conference on Marine Structures (MARSTRUCT 2017), pp. 405-413, 2017.

[2] Brochard, K., Le Sourne, H. \& Barras, G., Extension of the string-on-foundation method to study the shock wave response of an immersed cylinder. International Journal of Impact Engineering, 117, pp. 138-152, 2018. https://doi.org/10.1016/j.ijimpeng.2018.03.007 
[3] Wierzbicki, T. \& Hoo Fatt, M.S., Damage assessment of cylinders due to impact and explosive loading. International Journal of Impact Engineering, 13(2), pp. 215-241, 1993. https://doi.org/10.1016/0734-743x(93)90094-n

[4] Gupta, S., Matis H., LeBlanc, J.M. \& Shukla, A., Shock initiated instabilities in underwater cylindrical structures. Journal of the Mechanics and Physics of Solids, 95, pp. 188-212, 2016. https://doi.org/10.1016/j.jmps.2016.05.034

[5] Biglarkhani, M. \& Sadeghi, K., Incremental explosive analysis and its application to performance-based assessment of stiffened and unstiffened cylindrical shells subjected to underwater explosion. Shock and Vibration, 2017, pp. 1-17, 2017. https://doi.org/10.1155/2017/3754510

[6] Wierzbicki, T. \& Suh, M.S., Indentation of tubes under combined loading. International Journal of Mechanical Sciences, 30(3-4), pp. 229-248, 1988. https://doi.org/10.1016/0020-7403(88)90057-4

[7] Cole, R.H., Underwater Explosions, Dover Publications, New York, NY, 1965.

[8] Geers, T.L., Doubly asymptotic approximations for transient motions of submerged structures. Journal of Acoustical Society of America, 64(5), pp. 1500-1508, 1978. https://doi.org/10.1121/1.382093

[9] Taylor, G.I., The pressure and impulse of submarine explosion waves on plates. Underwater Explosion Research (Office of Naval Research, Washington D. C., 1950), 1, pp. 1155-1174, 1941.

[10] Pinna, R. \& Ronalds, B., Hydrostatic buckling of shells with various boundary conditions. Journal of Constructional Steel Research, 56(1), pp. 1-16, 2000.

https://doi.org/10.1016/s0143-974x(99)00104-2 